\title{
Mitigation of redundant handovers to femtocells by estimation of throughput gain
}

\author{
Zdenek Becvar* and Pavel Mach \\ Faculty of Electrical Engineering, Czech Technical University in Prague, Prague, Czech Republic
}

\begin{abstract}
A deployment of femtocells to mobile wireless networks can increase a throughput of indoor as well as outdoor users. On the other hand, it introduces several problems such as serious interference or high number of performed handovers. This paper is focused on mitigation of redundant handovers to femtocells with open or hybrid access. The redundant handovers decrease user's throughput due to a management overhead and due to introduced interruption. We design a novel handover decision algorithm based on an estimation of throughput gain reached by a handover to a femtocell. In the proposal, the handover is initiated only if the estimated gain in user's throughput exceeds a predefined threshold. As the results indicate, high ratio of eliminated redundant handovers is achieved by the designed procedure. Moreover, a drop in user's throughput due to the handover procedure is reduced by the proposed algorithm and thus the user's throughput is increased.
\end{abstract}

Keywords: Estimated throughput gain, femtocell, handover, mobility, small cells

\section{Introduction}

Femtocells are deployed to improve signal quality in buildings or to offload a Macro Base Station (MBS). The femtocell is represented by a Femto Access Point (FAP), which is a simplified MBS located mostly in a user's premise or an office. The FAP is characterized by low transmitting power, low cost, and a backbone connection usually ensured through a wired link (e.g., xDSL, cable, optical fiber, etc.).

Three types of user's accesses can be provided by the FAP: open, closed, and hybrid. In case of the open access, all users under coverage of the FAP can connect to it. A benefit of the open access consists in an opportunity to offload the MBS by serving several outdoor users in areas with heavy traffic load or by serving users far from the MBS [1]. On the contrary, the FAP with the closed access admits only users included in so-called Closed Subscriber Group (CSG) list. The interference should be carefully managed in areas with dense deployment of the FAPs to minimize system performance degradation in case of the closed access. The hybrid access is a combination of both. It means, a part of FAP's transmission capacity is dedicated for the CSG users and the rest of the bandwidth can be shared by other users. As presented in [2], the open access provides higher mean throughput experienced by users when compared to the closed one for low densities of user. However, closed access is preferred for high density of the MBS's users [3].

A subscriber can deploy the FAP anywhere in his/her premises and he/she can turn it on and off anytime. It leads to several problems related to Radio Resource Management (RRM). To exploit an

\footnotetext{
${ }^{*}$ Corresponding author: Zdenek Becvar, Faculty of Electrical Engineering, Czech Technical University in Prague, Technicka 2, 16627 Prague, Czech Republic. Tel.: +420 22435 5994; Fax: +420 23333 9810; E-mail: zdenek.becvar@ fel.cvut.cz.
} 
advantage of open (or hybrid) access simultaneously with ensuring unaffected Quality of Service (QoS) for mobile users attached to the MBS, the handover procedure must be handled as efficiently as possible. By efficient handover is meant a fast handover that is not initiated redundantly but only in cases when it is necessary to provide required QoS or to improve the network performance. In a network with femtocells, three types of handover can be distinguished: hand-in (handover from the MBS to the FAP), hand-out (handover from the FAP to the MBS), and inter-FAP (handover between two FAPs) (for more details see, e.g., [4]).

To improve the network performance, a coordination between all involved FAPs and MBSs via backbone can be established. The coordination is understood as an exchange of additional control and status information between network entities (FAPs and MBSs) via backbone. This approach guarantees more efficient RRM [3,5]. On the other hand, the coordination consumes a part of backbone capacity. Hence, it decreases the backbone capacity available to all users of the FAP.

This paper provides a solution for efficient elimination of redundant handovers in networks with FAPs if advantage of the coordination among the MBSs and the FAPs via backbone is exploited. The proposed solution replaces a conventional handover decision algorithm based on a simple comparison of the signal levels. The designed handover is based on an estimation of throughput gain acquired by performing handover to the FAP. Therefore, this approach is further denoted as ETG handover (Estimation of Throughput Gain for handover decision). The gain in throughput is derived from the estimated evolution of the signals levels of all involved cells measured by the User Equipment (UE) and from an estimated time spent by the users in the FAP. This approach is applicable to hand-in since estimation of the time spent in cells with low radius is significantly more precise than in case of large cells [6]. Therefore, this approach is not limited to femtocells only but it is applicable also for cells such as picocells or microcells, known as small cells.

The rest of the paper is organized as follows. The next section gives an overview on related work and state of the art in the field of mobility support for femtocells. The third section introduces the novel handover decision procedure including definition of all network and system assumptions, and procedures for determination of all required parameters. Evaluation methodology, network deployment, system parameters, and results of evaluations and simulations are presented in Section 4. The last section summarizes the main conclusions and indicates possible directions for future work.

\section{Related work}

Several techniques such as Hysteresis Margin (HM) [7,8] or windowing (also known as signal averaging) [7] are defined to eliminate redundant handovers in conventional networks without the FAPs. In case of using any technique for elimination of redundant handovers a drop in throughput is introduced. This is due to a short time when the UE communicates with the serving station even if the potential target station provides channel of a higher quality. A drop in throughput is even more significant if conventional techniques (e.g., HM or windowing) are utilized for elimination of the redundant handovers in scenario with the FAPs [9]. A modification of the conventional HM is defined in [10]. The authors evaluate so-called adaptive HM in scenario with deployed MBSs but without the FAPs. An enhancement of the adaptive HM applicable to the networks with the FAPs is further introduced in [11]. The authors propose to use CINR (Carrier to Interference plus Noise Ratio) levels for adaptation of the actual value of HM. This approach improves users' throughput; however, it does not increase the amount of eliminated handovers. 
The handover mechanism for the FAPs considering an asymmetry of transmitting power of the FAP and the MBS is introduced in [12] and further extended in [13]. This mechanism compares the level of the average signal received from the potential target FAP with the absolute threshold value of $-72 \mathrm{~dB}$. Besides, the signal of the MBS is compared with a combination of the signals from the MBS and the FAP. After the comparison of individual results, either the MBS or the FAP is selected as a serving station. This proposal increases the probability of handover to the FAP if this FAP provides the signal above the threshold and if the FAP is deployed far from the MBS. Otherwise, if the threshold is not met, the handover is performed as in the conventional way. Unfortunately, the paper provides no solution for scenario with overlapping femtocells. As the authors indicate, the proposed algorithm mitigates redundant handovers if the FAP is close to the MBS. However, overall amount of handovers is even increased comparing to the conventional approach. The authors also do not consider limited capacity of FAP's backbone in their evaluations.

A combination of additional parameters, such as user's speed and QoS requirements, for improvement of the handover decision is presented in [14]. Although the number of unnecessary handovers is reduced, user's throughput is also negatively influenced. The speed-aware algorithm is proposed also in [15]. The authors exploit a fuzzy-logic system for the handover decision. The similar idea is further elaborated and extended in [16] where a new fuzzy-logic based handover algorithm with awareness of the speed is introduced. However, both papers are focused only on conventional networks and femtocells specifics are not considered.

Another approach eliminating redundant handovers is to adapt the transmission power of the FAPs. The proposals dealing with the power control adjustment to reduce the number of redundant handovers in femtocells are presented, e.g., in [17-19]. All these proposals eliminate majority of the redundant handovers. Nevertheless, the advantage of throughput gain due to utilization of the open/hybrid access (illustrated in [20]) is also distinctively suppressed by a reduction of the FAP's transmitting power. Therefore, these solutions are more suitable for closed access femtocells.

The authors of [21] discuss vertical handover between IEEE 802.16e and WLAN to maximize user's satisfaction. Taking lower cost of connection via WLAN into account, the authors suggest keeping the user connected to WLAN if it provides sufficient capacity to the user. However, handover decision based only on current bit rate achieved by the UE leads to the redundant handovers if WLAN's load fluctuates frequently. Moreover, the authors assume invariable throughput for users no matter what is its relative position with respect to the MBSs and WLAN access points. It means a variability of throughput in dependence on the distance between user and its serving and interfering nodes is not considered.

Prediction-based algorithms can be exploited for handover to improve its efficiency (see, e.g., [2224]). The prediction-based approaches reach high efficiency in determination of the target MBS. Nevertheless, by deployment of the FAPs, the prediction accuracy is strongly affected since the FAP's radius is very low and it overlaps with the MBSs. Moreover, even if the prediction reaches high efficiency in term of a high ratio of correctly predicted target cells, the handover to the estimated target cell can be inefficient if this cell is the FAP. This is due to a short time spent by the UE under the FAP's coverage or due to limited capacity of the FAP's backbone.

In this paper, we propose the algorithm for handover decision based on a profitability of the handover to the FAP. The handover is performed only if an estimated throughput offered to a UE by the FAP exceeds the throughput offered by the MBS. Both radio as well as backbone parameters of the FAPs and the MBSs are taken into account in handover decision. Consequently, the proposed procedure rejects only those handovers to the FAPs that do not introduce any considerable improvement in users' connection. In other words, the purpose of the proposed handover is to reduce amount of initiated handovers to the FAPs with low profit (or even with loss) for either network (operator) or users. 
Table 1

Notation of parameters used for description of the ETG handover algorithm

\begin{tabular}{|c|c|}
\hline Symbol & Definition \\
\hline$t_{c}, k_{c}$ & $\begin{array}{l}\text { Time in Cell. Mean time spent by users in the cell expressed as a time interval and a number of } \\
\text { signal level samples respectively; } t_{c}=\left(k_{c}-1\right) \times t_{s} \text {, where } t_{s} \text { is a channel quality measurement and } \\
\text { reporting period. }\end{array}$ \\
\hline$k_{H O, \text { in }}, k_{H O, \text { out }}$ & $\begin{array}{l}\text { Index of signal samples respective to the time instant of the handover to the FAP decision }\left(k_{H O \text {,in }}\right) \text { and } \\
\text { of hand-out from the FAP }\left(k_{H O \text {,out }}\right) \text {. }\end{array}$ \\
\hline$\overline{s_{b, a v g}}, \overline{s_{f, a v g}}$ & $\begin{array}{l}\text { Estimated mean values of the signals received from the MBS and the FAP in the time interval } k \in \\
\left\langle k_{H O, \text { in }}, k_{H O \text { out }}\right\rangle \text {. }\end{array}$ \\
\hline$C_{F A P}$ & Maximum capacity of the FAP available for outdoor user's limited by the backbone. \\
\hline$d_{U E, t_{c}}$ & Data prepared for a transmission by the UE during $t_{c}$. \\
\hline$g_{\mathrm{HO}}$ & Real gain in a signal level due to performing handover to the FAP. \\
\hline$g_{\mathrm{HO}, \text { est }}$ & Estimated gain in signal level due to performing handover to the FAP. \\
\hline$T_{B S, e s t}, T_{F A P, e s t}$ & $\begin{array}{l}\text { Estimated transmission rate of the UE if it stays connected to the MBS and if it performs handover to } \\
\text { the FAP respectively. }\end{array}$ \\
\hline$G_{H O, e s t}$ & Throughput gain without consideration of $C_{F A P}$ and $d_{U E, t_{c}}$. \\
\hline$T G_{H O, e s t}$ & Throughput gain taking $C_{F A P}$ and $d_{U E, t_{c}}$ into account. \\
\hline$\gamma_{T h r}$ & Relative threshold for ETG handover initiation. \\
\hline$b^{\prime} s_{s}$ & Current bit rate experienced by the UE at the serving station. \\
\hline$m_{T h r}$ & Multiplier of $b p s_{s}$ to determine $\gamma_{T h r} ; \gamma_{T h r}=m_{T h r} \times b p s_{s}$ \\
\hline$n_{\text {conn }}^{\min }$ & $\begin{array}{l}\text { Minimum amount of connections to the FAP that has to be performed before utilizing ETG for han- } \\
\text { dover decision. }\end{array}$ \\
\hline
\end{tabular}

\section{Handover based on estimation of throughput gain}

The principle of the ETG is based on a comparison of an evolution of the signals received by the UE from cells involved in handover. Furthermore, a time spent by the users under coverage of the FAPs must be derived. An implementation of those principles to the networks involves several assumptions and requirements summarized in the next subsection.

\subsection{Notation and assumptions}

To easy follow the explanation of the ETG procedure, the most important parameters used in description of the ETG are shown in Table 1.

For implementation of our proposal, several assumptions are defined:

- Assumption I: Coordination between the MBS and the FAP for purpose of exchanging FAP's backbone load and status information is enabled. Therefore, an overhead generated due to the coordination must be considered. The amount of generated overhead is discussed later in this paper in Section 4.4.

- Assumption II: The area covered by the FAPs is in order of tens of meters. This is common assumption and it is implied by low transmitting power of the FAPs. Therefore, this assumption is fulfilled in all cases.

- Assumption III: Only pedestrians can perform handover to the FAPs since fast moving users (e.g., vehicular users) do not spend enough time in the femtocell to take any advantage of hand-in. This is also a common assumption in the femtocell networks (see, e.g. [14]). If the vehicular users enter the area of the FAP, the handover will be performed only on the condition that the connection would be dropped due to unsatisfactory channel quality. Otherwise, the connection to the current serving cell is kept. To decide whether the user is pedestrian or not, a user's speed must be determined. For this purpose, either GPS or a localization based on propagation of the transmitted signal (e.g., 
multilateration [25]) can be used. An advantage of GPS is its higher accuracy. On the other hand, GPS increases UE's power consumption.

\subsection{General principle of the ETG handover}

The principle of the proposed ETG handover can be explained as follows. Let $s_{b}(k)$ and $s_{f}(k)$ represent the signal levels of the MBS and the FAP respectively. Both signals are obtained by a periodic measurement and reporting of the signals transmitted by the MBS and the FAP. The signal level received by a UE is influenced by a transmitting power of the MBS (denoted as $P_{b, T x}$ ) and the FAP (denoted as $\left.P_{f, T x}\right)$, by path losses $\left(P L_{b}, P L_{f}\right)$, and by random effects such as shadowing, fast fading, or measurement errors expressed, in summary, by parameter $u_{b}(k)$ for the MBS and $u_{f}(k)$ for the FAP. Thus, the signal levels can be defined as:

$$
\begin{aligned}
& s_{b}(k)=P_{b, T x}-P L_{b}(k)-u_{b}(k) \\
& s_{f}(k)=P_{f, T x}-P L_{f}(k)-u_{f}(k)
\end{aligned}
$$

To eliminate random effects influencing signal levels at the UE, the signal averaging is assumed. Rectangular window $w(k)=1$ for $k \in\left(i, i-n_{w}\right)$ is considered in this paper. Parameter $n_{w}$ represents the length of the window. The signal levels used by the UE for the handover decision are obtained according to the next formulas:

$$
\begin{aligned}
& \overline{s_{b}}(k)=s_{b}(k) * w(k) \\
& \overline{s_{f}}(k)=s_{f}(k) * w(k)
\end{aligned}
$$

The conventional handover decision is based on a comparison of the signal levels received from a potential target station $\left(\overline{s_{t}}(k)\right)$ with the signal level received from a serving station $\left(\overline{s_{s}}(k)\right)$, i.e., the handover is performed if:

$$
\overline{s_{t}}(k)>\overline{s_{s}}(k)+\Delta_{H M}
$$

where $\Delta_{H M}$ represents the hysteresis margin. Signal levels $\overline{s_{s}}(k)$ and $\overline{s_{t}}(k)$ correspond either to $\overline{s_{b}}(k)$ or to $\overline{s_{f}}(k)$ depending on a type of handover as follows:

- $\overline{s_{s}}(k)=\overline{s_{b}}(k)$ and $\overline{s_{t}}(k)=\overline{s_{f}}(k)$ for hand-in;

- $\overline{s_{s}}(k)=\overline{s_{f}}(k)$ and $\overline{s_{t}}(k)=\overline{s_{b}}(k)$ for hand-out;

- $\overline{s_{s}}(k)=\overline{s_{f}}(k)$ and $\overline{s_{t}}(k)=\overline{s_{f}}(k)$ for inter-FAP handover.

In the proposed ETG handover procedure, we define a general condition for the handover initiation as:

$$
g_{H O}>g_{T h r}
$$

where $g_{H O}$ is the overall profit in signal level achieved by the handover to the FAP and $g_{T h r}$ is a predefined threshold for the handover initiation. The profit $g_{H O}$ is proportional to the area limited by $\overline{s_{b}}(t)$ and $\overline{s_{f}}(t)$ from the time instant $t_{H O, \text { in }}$ till $t_{H O, \text { out }}$, as depicted in Fig. 1.

The gain $g_{H O}$ is defined by subsequent equation:

$$
g_{H O}=\int_{t_{H O, \text { in }}}^{t_{H O, \text { out }}}\left(\overline{s_{f}}(t)-\overline{s_{b}}(t)\right) d t
$$




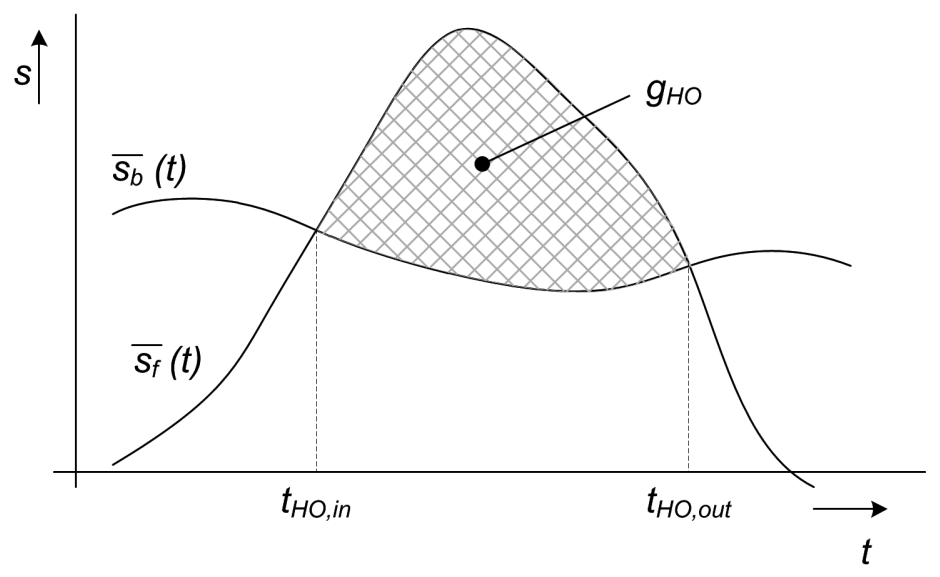

Fig. 1. Gain in signal level due to handover from a MBS to a FAP.

The user's gain, as the result of the handover, can be expressed also for the situation when discrete signal samples are obtained by periodic measurements:

$$
g_{H O}=\sum_{i=k_{H O, \text { in }}}^{k_{H O, \text { out }}}\left(\overline{s_{f}}(i)-\overline{s_{b}}(i)\right)
$$

where $k_{H O, \text { in }}$ and $k_{H O, \text { out }}$ correspond to the indexes of signal samples obtained in $t_{H O, \text { in }}$ and $t_{H O, \text { out }}$ respectively.

\subsection{Determination of gain caused by a handover to FAPs}

Parameters $\overline{s_{b}}(k), \overline{s_{f}}(k), k_{H O \text {,in }}$, and $k_{H O \text {,out }}$ must be found to determine $g_{H O}$. Parameters $k_{H O \text {,in }}$ and $k_{H O \text {,out }}$ represent the instants of the UE's entering and leaving the FAP respectively. In fact, the knowledge of $k_{H O \text {,in }}$ and $k_{H O \text {,out }}$ is not necessary. Only the difference between both instances, $k_{c}=k_{H O \text {,out }}-k_{H O \text {,in }}$, is sufficient to be determined. In praxis, the parameter $k_{c}$ represents a mean time spent by the users in the FAP's cell and it is expressed as an amount of signal measurement periods (sampling period) during which the UE is connected to the FAP.

An inaccuracy of determination of $k_{c}$ can be caused by different movement of users in the cell and by the variable speed of users. Considering assumption II, the estimation of the throughput gain is distinctively more precise for handover to the FAP comparing to the handover among MBSs since the difference between minimum and maximum times spent in the cell varies only slightly comparing to the MBSs as demonstrated in [6]. Therefore, the proposed algorithm is applicable for hand-in. For hand-out or for handover between the MBSs, the conventional handover decision is used.

Once $k_{c}$ is derived, an estimation of the MBS's and the FAP's signal levels progress must be done. The estimation means a determination of $\overline{s_{b}}(k)$ and $\overline{s_{f}}(k)$ in the interval $k \in\left(k_{H O \text {,in }}, k_{H O \text {,out }}\right)$. The precise estimation of $\overline{s_{b}}(k)$ and $\overline{s_{f}}(k)$ over the whole interval $k \in\left(k_{H O, \text { in }}, k_{H O, \text { out }}\right)$ is very complicated since both signal levels are influenced by many random factors. For the sake of computational complexity lowering, we propose to estimate the mean signal levels received by the UE in the interval $k \in\left(k_{H O \text {,in }}, k_{H O \text {,out }}\right)$ from the MBS and the FAP. The mean levels of signals are denoted as $\overline{s_{b, a v g}}$ and $\overline{s_{f, a v g}}$. An inaccuracy of the signal level estimation can be compensated by the selection of proper threshold $g_{T h r}$ for performing 
handover to the FAP and by its re-adjustment if more or less handovers to the FAPs are required as explained later in Section 3.4. For the estimation of the average levels, we consider a simple extrapolation of $\overline{s_{b}}(k)$ as defined by the following formula:

$$
\overline{s_{b, a v g}}=s_{b}\left(k_{H O}\right)+\Delta_{s_{b}} ; \Delta_{s_{b}}=\frac{k_{c}}{2 \times i_{\max }} \sum_{i=i_{\min }}^{k_{H O, \text { in }}}\left[\left(s_{b}(i+1)-s_{b}(i)\right) \times\left(\frac{i}{i_{\max }-1}\right)\right]
$$

where $i_{\max }$ is the number of samples considered for the extrapolation; and $i_{\min }=k_{H O \text {, in }}-\left(i_{\max }-1\right)$. Of course, advanced derivation of the average levels can be employed to improve performance of the ETG. However, our goal is to show efficiency of the ETG even with a simple estimation in this paper

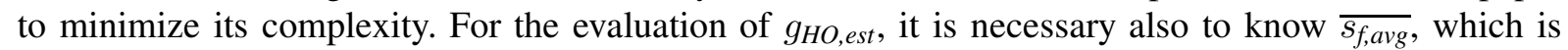
calculated in the same way as $\overline{s_{b, a v g}}$. If both estimated signal levels and $k_{c}$ are known, the estimated gain $g_{\mathrm{HO}, \text { est }}$ is derived as:

$$
g_{H O, e s t}=f_{T}\left(t_{c} \times\left(\left(\overline{s_{f, a v g}}+I_{f}\right)-\left(\overline{s_{b, a v g}}+I_{b}\right)\right)\right)
$$

where $f_{T}$ represents the transformation function for selection of appropriate Modulation and Coding Scheme (MCS) according to the received signal levels (see, e.g., [26]), $I_{f}$ and $I_{b}$ express interference in case if the FAP and the MBS are the serving cells respectively. In the estimation process, the prediction of interfering signals is not considered since it would lead to a need for the estimation of a large amount of signals. Such a complex prediction of a large number of signals introduces high susceptibility to inaccuracies. Moreover, high computational requirements are imposed by the prediction of interference. To lower complexity of the estimation process, the interference observed at the time of the handover decision is considered in Eq. (8). Note that the error introduced by this approach is not significant since the interference (either $I_{f}$ and $I_{b}$ ) is imposed by all cells in the UE's vicinity and the set of interfering cells for $I_{f}$ and $I_{b}$ differs only in inclusion of the target FAP or the serving MBS. An estimation of interference can potentially further improve performance of ETG. Therefore, the assumed way of consideration of interference represents the worst-case situation from the ETG performance point of view but the complexity of the estimation is minimized.

So far, a limitation of the FAP's backbone capacity was not considered for the estimation of the gain in signal level $\left(g_{H O, e s t}\right)$. Moreover, the handover should be performed only if the UE has data to be sent during the connection to the FAP. Otherwise, the handover is not necessary. To incorporate both limiting factors to ETG, $g_{H O, e s t}$ must be translated to a gain in user's throughput $\left(G_{H O, e s t}\right)$ according to the next formula:

$$
G_{H O, e s t}=\left(k_{c} \times\left(T_{F A P, e s t}-T_{B S, e s t}\right)\right)
$$

Both $T_{F A P, e s t}$ and $T_{B S, e s t}$ (see Table 1 for the explanation of those parameters) are derived from $\overline{s_{f, a v g}}$ and $\overline{s_{b, a v g}}$ based on MCS (e.g., according to [26]).

The final estimated throughput gain with respect to the backbone limitation and user's data is expressed by the following equation:

$$
T G_{H O, e s t}=\min \left(C_{F A P}, d_{U E, t_{c}}, G_{H O, e s t}\right)
$$

Parameters $C_{F A P}$ and $d_{U E, t c}$ are explained in Table 1. 
According to assumption I, the information on the available capacity of the FAP's backbone should be exchanged between the FAPs and the MBS. This information is delayed due to transmission via the FAPs backbone, which is of a lower quality than the backbone of the MBSs. The delay is supposed to be up to tens of milliseconds, which corresponds to the typical end-to-end packet delay for ADSL link [27]. Taking into account assumption III (i.e., only pedestrians are admitted to the FAPs), the delay of tens of milliseconds leads to only negligible shift in users' position (tens of centimeters). Hence, the channel conditions can be considered as stationary during this very short period. The delay only slightly postpones the decision on handover and the estimation of throughput gain is affected insignificantly.

\subsection{ETG handover decision}

Once the $T G_{H O \text {,est }}$ is derived, it is compared with a relative ETG handover threshold $\left(\gamma_{T h r}\right)$. The threshold $\gamma_{T h r}$ is related to the actual bit rate of the UE $\left(b p s_{s}\right)$ and it is expressed as the multiple $\left(m_{T h r}\right)$ of the current bit rate experienced by the UE at the serving MBS. This is defined by the following equation:

$$
T G_{H O, e s t}>\gamma_{T h r}=b p s_{S} \times m_{T h r}
$$

The $\gamma_{T h r}$ is used for the elimination of hand-ins that offers only low to moderately higher throughput than the serving station. In such cases, the handover is not profitable due to a short break in user's connection and additional signaling overhead introduced by the handover management.

In real networks, an inaccuracy in estimation of $T G_{H O \text {,est }}$ is expected. The level of over/underestimation of $T G_{H O \text {,est }}$ in real networks is proportionally the same for all FAPs and MBSs as it is calculated in the same way for all of these entities. Thus, the over/under-estimation of $T G_{H O \text {,est }}$ can be reduced by re-adjustment of $\gamma_{T h r}$ if more/less handovers to the FAPs are desirable, e.g., for the purpose of the MBS's offloading.

The evaluation of the ETG handover conditions can be performed either once when the conventional handover conditions, expressed in Eq. (3), are met for the first time or continuously during whole operation of the UE. In our proposal, the evaluation of the ETG conditions is performed continuously. This way, an impact of rapid channel variations and the inaccuracy in signal levels estimation are reduced since these phenomena just postpone the handover decision for a certain time. In order to avoid negative affection of the accuracy of $T G_{H O \text {,est }}$ by postponing the handover due to both factors, a temporary $k_{c, t}$ is used for derivation of $T G_{H O, e s t}$. The $k_{c, t}$ is obtained from $k_{c}$ by subtraction of a time interval elapsed since the conventional handover conditions are fulfilled.

In the case of the UE entering the area where more FAPs meet the conditions for the handover initiation, i.e., more FAPs fulfill Eq. (4), the FAP with maximum $T G_{H O}$,est is selected as the target one. If no FAP fulfils ETG handover condition defined in Eq. (11), the MBS is selected as the target station. If the UE enters the location with more possible target stations (usually at the beginning of simulation or network operation) before accurate $k_{c}$ for each FAP in the area is set up, the selection of the target station is based on the conventional handover. Since the FAPs are partially controlled by users, an event such as occasional FAP's turn-on/off should be addressed. In this case, the backbone is used to inform the MBS and all adjacent FAPs of the change in neighbor cell list. All adjoining FAPs should reinitialize the evaluation of $k_{c}$. Nevertheless, this event is assumed to appear very rarely. 


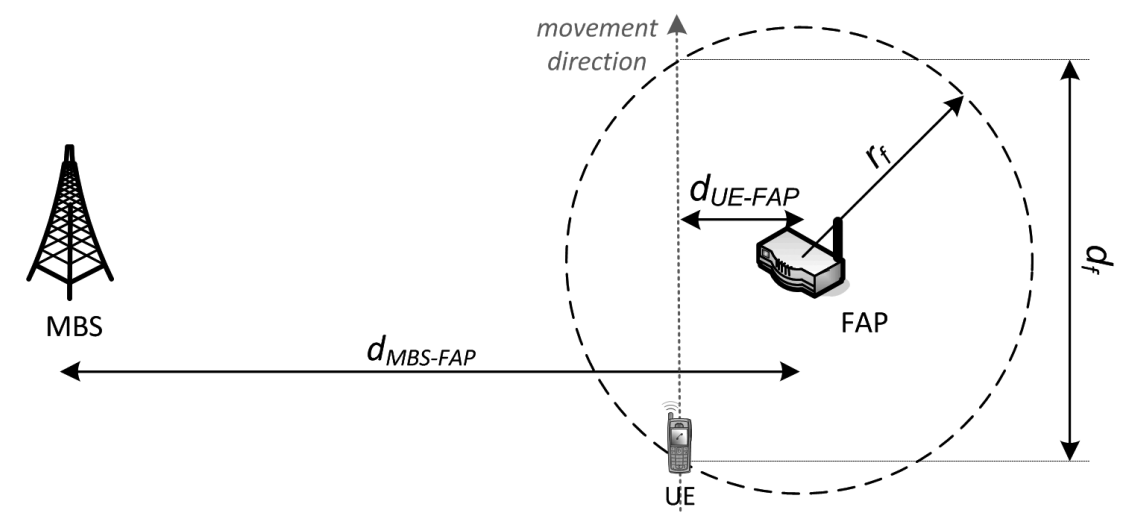

Fig. 2. Deployment for performance evaluation.

\section{Evaluation of ETG performance}

First, the performance of the ETG is evaluated under the assumption of perfect knowledge of all required parameters in simple scenario with one MBS and one FAP. Further, an impact of imperfect determination of $k_{c}$ is evaluated under the same scenario. To prove the algorithms efficiency in real networks, system level simulations in MATLAB are performed as well. Both ways of the evaluation use common models, deployments, and simulation methodology recommended by Small Cell Forum for evaluation of femtocells. An impact of the ETG on the backbone communication is discussed as well in this section.

\subsection{Evaluation of ETG performance for perfect knowledge of $k_{c}$}

For evaluation of the ETG under the assumption of perfect knowledge of all parameters and signal propagation characteristics, a MBS and a FAP are deployed in mutual distance $d_{M B S-F A P}$ as depicted in Fig. 2. The users are moving along a straight line with random distance from the FAP, denoted as $d_{U E-F A P}$. The distance $d_{U E-F A P}$ represents the shortest distance between the UE's movement and the FAP during a simulation drop. The performance is evaluated for $d_{M B S-F A P}$ varying in range from $100 \mathrm{~m}$ to $400 \mathrm{~m}$ in step of one meter and then the results are averaged out. For each $d_{M B S-F A P}$, sixty simulation drops with random user's speed, ranging between 0.97 and $1.74 \mathrm{~m} / \mathrm{s}$ [28], are performed. The distance $d_{U E-F A P}$ is equally distributed for each $d_{M B S}-F A P$.

The outdoor users generate Constant Bit Rate (CBR) traffic during the evaluations. The amount of traffic load introduced by CBR is indicated in figures with results. Besides that, fixed indoor users generate load of $4 \mathrm{Mbps}$ for the FAP. The hybrid access with fifty percent of overall backbone capacity assigned for the indoor users is applied. The rest of the capacity is dedicated to the outdoor users. The full backbone capacity is 8 Mbps.

Transmitting power of the MBS and the FAP is set to the fix value of $46 \mathrm{dBm}$ and $15 \mathrm{dBm}$ respectively. Signal level experienced by the UEs from the MBS is determined according to Okumura-Hata path loss model for outdoor to outdoor communication as suggested by Small Cell Forum [29]. The channel between the UE and the FAP is modeled according to ITU-T R.1238 path loss model for one floor house [29]. The FAP's path loss model includes also a wall with attenuation of $10 \mathrm{~dB}$. The shadowing with standard deviation of $8 \mathrm{~dB}$ (for the MBS) and $4 \mathrm{~dB}$ (for the FAP) is also taken into account. 
Table 2

System parameters for evaluations

\begin{tabular}{ll}
\hline Parameter & Value \\
\hline Carrier frequency & $2 \mathrm{GHz}$ \\
Transmitting power of MBS/FAP & $46 / 15 \mathrm{dBm}$ \\
Height of MBS/FAP/UE & $30 / 1 / 1.5 \mathrm{~m}$ \\
Channel bandwidth of MBS and FAP & $20 \mathrm{MHz}$ \\
Noise Power Spectral Density & $-174 \mathrm{dBm} / \mathrm{Hz}$ \\
Path loss model for MBS to UE (see [29]) & $69.55+26.26 \log (f)-13.82 \log \left(h_{B}\right)+\left(44.9-6.55 \log \left(h_{B}\right)\right) \log (d)-$ \\
& $(1.1 \log (f)-0.7) h_{M}+(1.56 \log (f)-0.8)$ \\
Path loss model for FAP to UE (see [29]) & $20 \log (f)+28 \log (d)-24$ \\
Wall Loss & $10 \mathrm{~dB}$ \\
Physical layer overhead & $25 \%[30]$ \\
Outdoor UE speed & $0.97-1.71 \mathrm{~m} / \mathrm{s} \mathrm{[28]}$ \\
\hline
\end{tabular}

To investigate throughput, the physical layer according to LTE-A release 10 using TDD with uplinkdownlink configuration " 1 " and special-subframe configuration " 0 " is implemented [30]. All major parameters for the evaluation are summarized in Table 2.

To determine $k_{c}$ for the purpose of the evaluation, knowledge of both user's speed and $d_{U E-F A P}$ is required. Knowing $d_{U E-F A P}$, the distance covered by a directly moving UE under the coverage of a FAP is:

$$
d_{f}=2 \sqrt{r_{f}^{2}-d_{U E-F A P}^{2}}
$$

where $r_{f}$ is the FAP's radius. The radius of the FAP is the distance between the FAP and the place where the handover is initiated. Analytically, it can be expressed as the distance where:

$$
\begin{aligned}
& s_{f}(k)=s_{b}(k)+\Delta_{H M} \\
& P_{f, T x}-P L_{f}(k)-u_{f}(k)=P_{b, T x}-P L_{b}(k)-u_{b}(k)+\Delta_{H M}
\end{aligned}
$$

Using transmitting power of the FAP and the MBS and both path loss models $P L_{b}, P L_{f}$ (see Table 2), the radius of the FAP can be derived, after several mathematical simplifications in Eqs (12) and (13), according to the next formula:

$$
r_{f}=\sqrt[35.04]{10^{\log _{10}\left(d_{M B S-F A P}^{28}\right)-27.65+\Delta_{H M}}}
$$

Then $t_{c}$ and $k_{c}$ is simply calculated as:

$$
\begin{aligned}
& t_{c}=d_{f} / v=2 r_{f} / v \\
& k_{c}=1+\left(t_{c} / t_{s}\right)
\end{aligned}
$$

First, the impact of $m_{T h r}$ on the amount of performed handovers and on the throughput of outdoor users is depicted in Figs 3 and 4 respectively. These figures show an impact of $m_{T h r}$ on the ETG performance. Therefore, all results in these figures are related to the maximum value obtained for individual level of the offered traffic, and there is no relation to other competitive handover techniques.

The amount of initiated handovers decreases with increase in $m_{T h r}$ until a minimum of performed handovers is reached. The minimum number of handovers is equal to the number of handovers that have to be performed since the signal from the MBS becomes of a very low quality and it would lead to loosing the connection of the UE to the network. In other words, if no handover would be performed in 


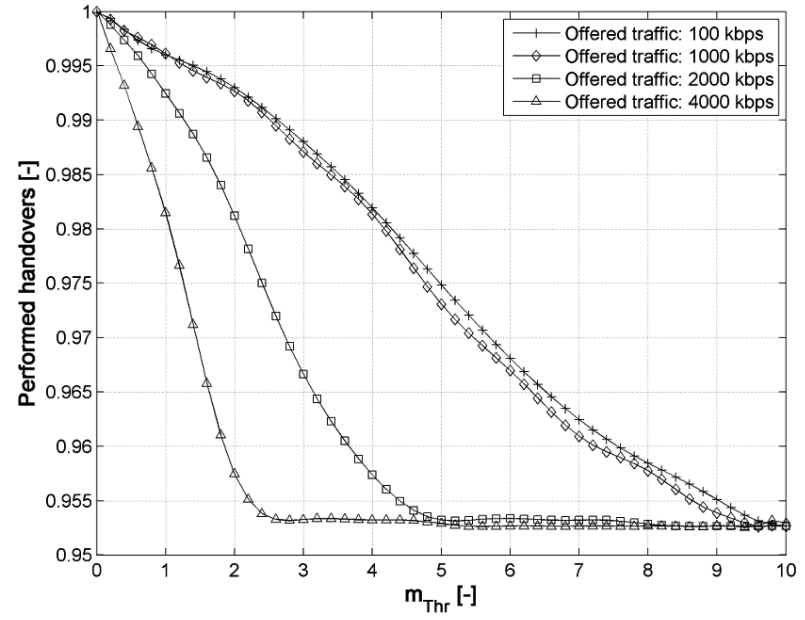

Fig. 3. Impact of $m_{T h r}$ on the amount of performed handover. Fig. 4. Impact of $m_{T h r}$ on the relative throughput of outdoor user.

this situation, the UE would not be able to communicate with the MBS due to high interference from the neighboring cells. As the results show, the amount of performed handovers depends not only on the ETG threshold value but also on the traffic offered by the outdoor UE. For higher offered traffic load, the lower multiplier of the current UE's bit rate, $m_{T h r}$, must be set up to reach maximum efficiency in elimination of the redundant handovers. This is since achievable gain in throughput is the multiplication of $m_{T h r}$ and current UE's bit rate, which is related to the offered traffic. By selection of the proper $m_{T h r}$, up to additional $5 \%$ of handovers can be eliminated comparing to the case of $m_{T h r}=0$.

An increase in $m_{T h r}$ leads to a drop in user's throughput that is, however, only negligible. Lowering the throughput is the cost of avoiding of the redundant handovers with low gain for users. This is due to a utilization of the channel, which is not of the best quality since the UE stays connected to the MBS although the signal from the FAP is better. Nevertheless, the impact of the ETG algorithm on mean throughput is only marginal (up to approximately $0.17 \%$ for $m_{T h r}=10$ and for $4000 \mathrm{kbps}$ of offered traffic).

\subsection{Impact of error in determination of $k_{c}$ on ETG performance}

So far, an exact estimation of $k_{c}$ based on perfect knowledge of all information according to Eqs (12)(15) was assumed. The perfect knowledge of $k_{c}$ is unrealistic for real networks. Therefore, an impact of an inaccuracy in determination of this parameter has to be evaluated. The inaccuracy is understood as an error in determination of $k_{c}$. It can be caused, for example, by movement of the UEs in different distances from the FAP or by variable speed of the users. The amount of performed handovers and the UE's throughput over the deviation of $k_{c}$ are illustrated in Figs 5 and 6 respectively. The $x$ axis represents maximum error in estimation of $k_{c}$ (denoted as $\zeta$ ) related to the calculated value obtained from Eq. (15). The individual error in $k_{c}$ is then defined by uniform distribution in interval $(-\zeta,+\zeta)$. Both figures show that high estimation error lowers the amount of performed handovers (additional 13\% of handovers are not performed comparing to perfect determination of $k_{c}$ ). This implies that the high $\zeta$ leads to the underestimation of the real gain in throughput and thus additional handovers are eliminated. Although this is at the cost of a drop in user's throughput, this drop is still very low. Even if the estimation error is up to $\pm 100 \%$, the relative throughput is decreased by additional roughly $0.85 \%$ comparing to the 


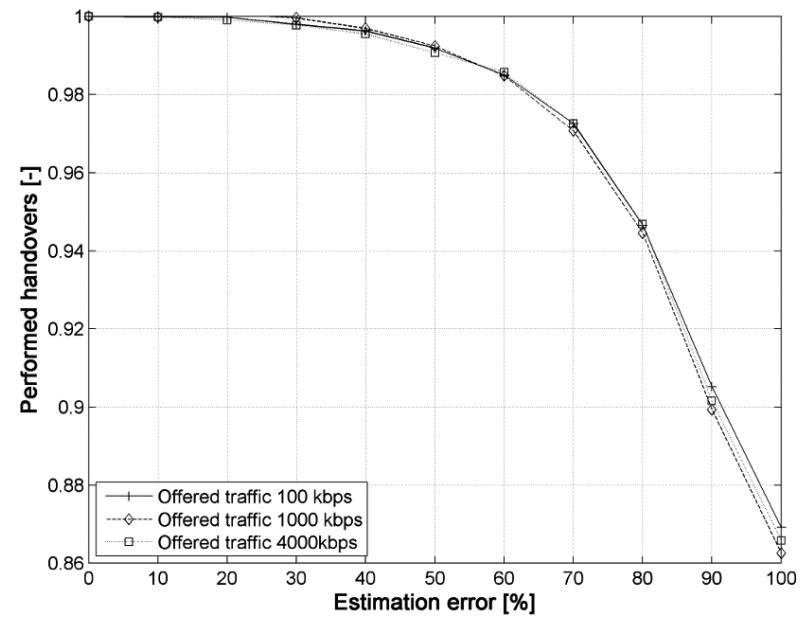

Fig. 5. Impact of error in estimation of $k_{c}$ on the amount of Fig performed handovers.

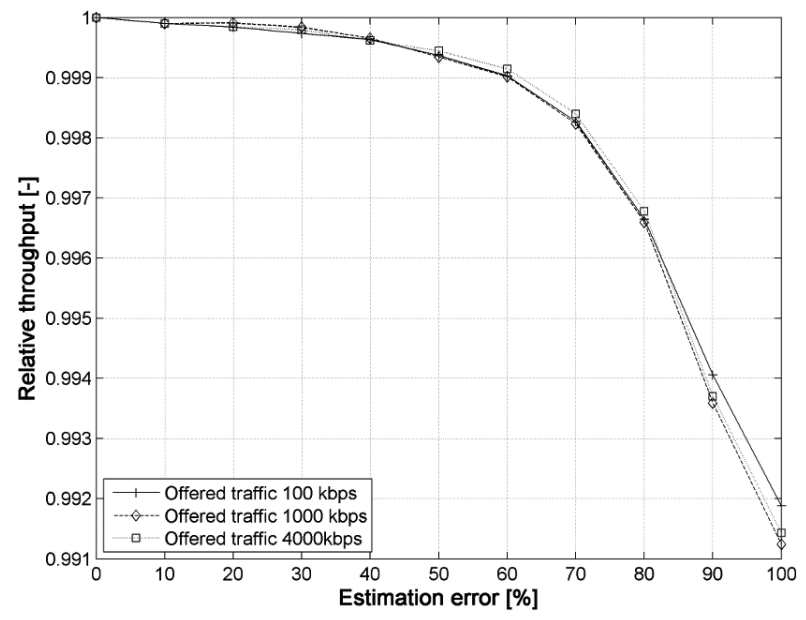

Fig. 6. Impact of error in estimation of $k_{c}$ on the throughput of users.

optimum determination of $k_{c}$ (see Fig. 6). Both Figs 5 and 6 further show that the impact of estimation error on throughput as well as on the amount of handovers is nearly independent on the offered traffic loads.

Figures 5 and 6 show that high error in estimation leads, on one hand, to a drop in throughput, however, it significantly improves efficiency in term of elimination of redundant handovers. To analyze how the performance is impaired or improved comparing to the conventional and competitive techniques, a system level simulations are performed.

\subsection{Evaluation of ETG performance by system level simulations}

Analytical evaluations show the impact of the $m_{T h r}$ determination on the performance of the ETG. As demonstrated in previous subsection, the performance can be influenced by selection of $m_{T h r}$ or by accuracy of determination of $k_{c}$ (as illustrated in Figs 5 and 6). Additionally, more UEs simultaneously connected to a FAP can influence the results due to limitation of FAP's backbone and due to interference. Therefore, system level simulations are performed. The results obtained by the simulations of ETG are confronted with other competitive algorithms: conventional hysteresis, adaptive hysteresis (described in [11]), and Moon's algorithm (presented in [12,13]). Other algorithms presented in Section 2 are focused on another aspects of handover, but do not target elimination of the redundant handovers or cannot be easily applied to environment with femtocells.

The simulations are performed for multiplied two stripes deployment [29] with $5 \times 5$ blocks of flats (see Fig. 7). The multiplication of two stripes deployment is used to fully exploit UEs mobility in the observed area. The FAPs density is equal to 2 FAPs per a block of 20 flats, i.e., $10 \%$ of flats are equipped with a FAP.

All UEs generate CBR traffic with randomly selected bit rate, which is selected for each UE according to lognormal distribution with mean of $500 \mathrm{kbps}(\mu=6.15, \sigma=0.35)$. The system models and parameters for simulation are in line with those used for analytical evaluation. The simulation duration is $10800 \mathrm{~s}$ of real-time with step of $1 \mathrm{~s}$.

Two parameters are monitored: drop in ratio of served traffic and ratio of eliminated handovers. The results observed from the simulations are summarized in Table 3. The results are related to the situation, 
Table 3

Comparison of performance of the ETG and competitive algorithms for $m_{T h r}=2$ (upper table) and $m_{T h r}=3$ (bottom table)

\begin{tabular}{lccrrr}
\hline Handover algorithm & \multicolumn{2}{c}{ Ratio of eliminated handovers [\%] } & & \multicolumn{2}{c}{ Drop in served traffic [\%] } \\
\cline { 2 - 3 } \cline { 5 - 6 } & wrt $\Delta_{H M}=0 \mathrm{~dB}$ & wrt ETG & & wrt $\Delta_{H M}=0 \mathrm{~dB}$ & wrt ETG \\
\hline Hysteresis; $\Delta_{H M}=3 \mathrm{~dB}$ & 21.90 & -51.69 & & 8.70 & 29.46 \\
Adaptive Hysteresis; $\Delta_{H M, \max }=3 \mathrm{~dB}$ & 21.88 & -51.73 & & 6.71 & -0.15 \\
Moon; $\varepsilon=0.05$ & 23.16 & -48.91 & & 20.57 & 206.10 \\
ETG; $\mathrm{m}_{T h r}=2$ & 45.33 & & & 6.72 & 0.00 \\
Hysteresis; $\Delta_{H M}=5 \mathrm{~dB}$ & 34.64 & -36.18 & & 14.57 & 14.45 \\
Adaptive Hysteresis; $\Delta_{H M, \max }=5 \mathrm{~dB}$ & 34.34 & -36.74 & 13.03 & 2.36 \\
Moon; $\varepsilon=0.05$ & 23.16 & -57.33 & & 20.57 & 61.59 \\
ETG; $\mathrm{m}_{T h r}=3$ & 54.28 & 0.00 & & 12.73 & 0.00 \\
\hline
\end{tabular}

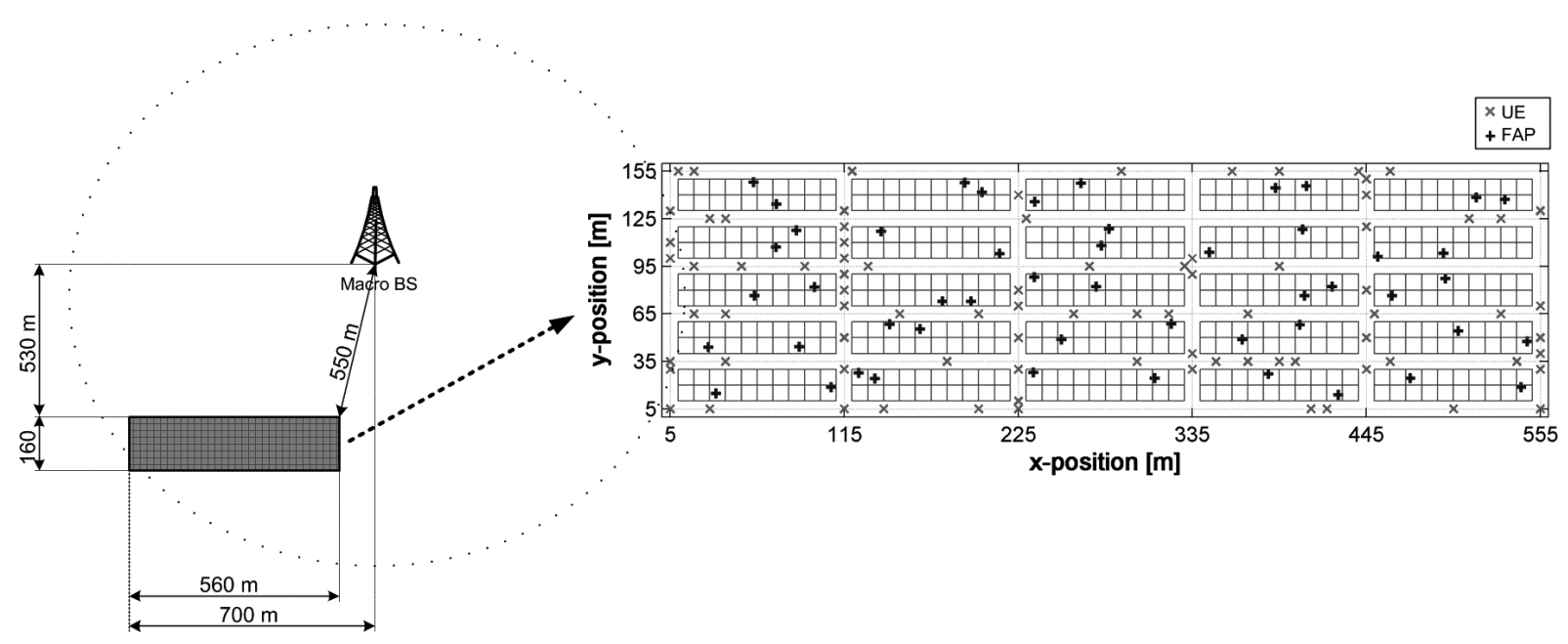

Fig. 7. Example of the simulation deployment for evaluation of ETG.

when no technique for the elimination of redundant handovers is used (i.e., $\Delta_{H M}=0 \mathrm{~dB}$ and each $\mathrm{UE}$ is connected to the best cell at each time). In other words, $0 \%$ of drop in the served traffic or $0 \%$ of eliminated handovers is the value reached by the simulation run with all techniques for handover elimination disabled. Further, a column showing how the performance is improved by comparison of the ETG with the competitive techniques (denoted as "wrt ETG") is included.

The results are split into two sub-tables according to the achieved performance to enable easier comparison of the results obtained for different setting of parameters. The constant levels of $m_{T h r}$ equal to 2 and 3 are selected since the ETG with those values reaches similar level of the drop in served traffic as the adaptive hysteresis. It enables easy comparison of all approaches. The same $m_{T h r}$ for all UEs disregarding offered traffic of the UEs represents the simplest way of handover management. On the other hand, it also slightly decreases the efficiency of the ETG. Assignment of individual $m_{T h r}$ for each UE according to its bit rate should slightly reduce overall amount of performed handovers as presented in Fig. 3. However, it is at the cost of more complex handover management. The levels of $\Delta_{H M}$ for the conventional and adaptive hysteresis are selected to follow values commonly used for those techniques. Higher values of $\Delta_{H M}$ lead to a significant decrease of users' throughput. On the other hand, lower values are not efficient for elimination of redundant handovers.

Adaptive hysteresis reaches roughly the same level of performed handovers as the conventional hysteresis for both levels of the $\Delta_{H M}$; however, the conventional hysteresis causes more significant drop 
in users' throughput. This confirms results presented in [11]. The proposed ETG can eliminate more handovers than both types of hysteresis while a lower decrease of throughput is ensured. If conventional hysteresis with $\Delta_{H M}=3 \mathrm{~dB}$ and ETG with $m_{T h r}=2$ are compared, the ETG eliminates roughly 2.07 times more $(45.33 \%$ instead of $21.9 \%)$ redundant handovers and it even reduces the drop in served traffic about $29.46 \%$. Comparing conventional hysteresis with $\Delta_{H M}=5 \mathrm{~dB}$ and ETG with $m_{T h r}=3$, the ETG eliminates 1.57 times more handovers while it reduces the drop in served traffic by $14.45 \%$. The drop in served traffic acquired by the ETG and the adaptive hysteresis is roughly the same for both with minor improvement on the side of the ETG. Nonetheless, the ETG eliminates more redundant handovers (2.07 and 1.58 times more handovers are eliminated by the ETG with $m_{T h r}=2$ and $m_{T h r}=3$ respectively). The Moon algorithm eliminates less handovers than the ETG for both $m_{T h r}$ (approximately $48.91 \%$ and $57.32 \%$ for $m_{T h r}=2$ and $m_{T h r}=3$ respectively) while it leads to higher drop in throughput than the ETG (rise by $206.10 \%$ and $61.59 \%$ for $m_{T h r}=2$ and $m_{T h r}=3$ respectively). To summarize the results, the ETG outperforms all competitive techniques in both observed parameters since it eliminates between at least 1.57 times more handovers while lower negative impact of the handover on the user's throughput is reached by ETG.

\subsection{Discussion of backbone overhead}

Relating to assumption I, the coordination among the FAPs and the MBSs via backbone must be established to implement the ETG. The coordination is used for exchange of information on the FAPs backbone status to determine maximum available backbone capacity for outdoor users. Only this information has to be delivered to the MBSs for the ETG purposes and it should be available at the MBS in a time instant of the handover decision. Therefore, the backbone status reporting interval should be similar to the reporting period of channel quality. In LTE, the channel quality reporting period can range between 2 and $160 \mathrm{~ms}$ [31]. Considering the worst case, the FAP's load must be reported each $2 \mathrm{~ms}$, i.e., 500 reports per second must be sent to the MBS. The size of the backbone load report should be in tens of bites as the report contains only indoor traffic load and the maximum backbone capacity. Therefore, the maximum overall backbone overhead of the ETG procedure is a couple of kbps in the worst-case scenario.

Further, an overhead can be generated due to the FAP's switch-on/off. For this purpose, only a message with the FAP's ID is delivered to neighboring FAPs to inform them about this event. Even if the amount of neighbors would be high (e.g., tens of FAPs), still the overhead in kilobits (tens of FAPs multiplied by tens of bits per message) is generated only very rarely since frequent turning-on/off the FAP cannot be expected. Both parts of the backbone overhead can be neglected considering the conventional backbone capacity in megabits.

\section{Conclusions}

This paper has introduced new algorithm for handover decision in femtocell networks with purpose to eliminate redundant handovers performed to the FAPs. The novel procedure is based on an estimation of UE's throughput gain acquired if a handover to the FAP is accomplished. This way, the handover to the FAP is performed only if the estimated gain in throughput exceeds a threshold related to the current throughput of the UE. The estimation of the gain in throughput is done according to the evolution of the levels of signals received by the UE from all involved stations. 
The results show high efficiency of the proposed ETG handover in elimination of redundant handovers. Comparing the proposed algorithm with other competitive algorithms, the proposed one eliminates additional between at least 57\% more of redundant handovers while the user's throughput can be even increased. The proposed handover implies only negligible additional signaling overhead transmitted by the FAP to the MBS via backbone. Note that the proposal can be applied not only to femtocells but also to all types of cells with low radius, i.e., small cell.

The currently proposed procedure can be further extended by more sophisticated prediction of signal evolution or by consideration of a signal level history for determination of throughput gain. In addition, the prediction of interference will be further investigated.

\section{Acknowledgments}

This work has been performed in the framework of the FP7 project FREEDOM IST-248891 STP, which is funded by the European Community. The Authors would like to acknowledge the contributions of their colleagues from FREEDOM Consortium (http://www.ict-freedom.eu).

\section{References}

[1] Small Cell Forum, Femtocells - Natural Solution of Offload, June 2010.

[2] D. Lopez-Perez, A. Valcarce, A. Ladanyi, G. de la Roche and J. Zhang, Intracell Handover for Interference and Handover Mitigation in OFDMA Two-Tier Macrocell-Femtocell Networks, EURASIP Journal on Wireless Communications and Networking, 2010, p. 15.

[3] P. Xia, V. Chandrasekhar and J.G. Andrews, Open vs. Closed Access Femtocells in the Uplink, IEEE Transactions on Wireless Communications 9(12) (2010), 3798-3809.

[4] L. Wang, Y. Zhang and Z. Wei, Mobility Management Schemes at Radio Network Layer for LTE Femtocells, In IEEE VTC Spring, 2009, pp. 1-5.

[5] S.P. Yeh, S. Talwar, S.C. Lee and H. Kim, WiMAX Femtocells: A Perspective on Network Architecture, Capacity, and Coverage, IEEE Communications Magazine 46(10) (2008), 58-65.

[6] Z. Becvar and P. Mach, On Enhancement of Handover Decision in Femtocells, In IFIP Wireless Days, 2011.

[7] M. Zonoozi, P. Dassanayake and M. Faulkner, Optimum Hysteresis Level, Signal Averaging Time and Handover Delay, In IEEE VTC, 1997, pp. 310-313.

[8] K.I. Itoh, S. Watanabe, J.S. Shih and T. Sato, Performance of handoff algorithm based on distance and RSSI measurements, IEEE Transactions on Vehicular Technology 51(6) (2002), 1460-1468.

[9] T. Jansen, I. Balan, J. Turk, I. Moerman and T. Kurner, Handover Parameter Optimization in LTE Self-Organizing Networks, In IEEE VTC Fall, 2010.

[10] S. Lal and D.K. Panwar, Coverage Analysis of Handoff Algorithm with Adaptive Hysteresis Margin, In ICIT, 2007, pp. 133-138.

[11] Z. Becvar and P. Mach, Adaptive Hysteresis Margin for Handover in Femtocell Networks, In ICWMC, 2010, pp. 256261.

[12] J.M. Moon and D.H. Cho, Efficient Handoff Algorithm for Inbound Mobility in Hierarchical Macro/Femto Cell Networks, IEEE Communications Letters 13(10) (2009), 755-757.

[13] J.M. Moon and D.H. Cho, Novel Handoff Decision Algorithm in Hierarchical Macro/Femto-Cell Networks, In IEEE WCNC, 2010, pp. 1-6, (2010).

[14] H. Zhang, X. Wen, B. Wang, W. Zheng and Y. Sun, A Novel Handover Mechanism between Femtocell and Macrocell for LTE based Networks, In ICCSN, 2010.

[15] L. Barolli, A speed-aware handover system for wireless cellular networks based on fuzzy logic, Mobile Information Systems 4(1) (2008), 1-12.

[16] G. Mino, L. Barolli, F. Xhafa, A. Durresi and A. Koyama, Implementation and performance evaluation of two fuzzybased handover systems for wireless cellular networks, Mobile Information Systems 5(4) (2009), 339-361.

[17] H. Claussen, F. Pivit and L.T.W. Ho, Self-Optimization of Femtocell Coverage to Minimize the Increase in Core Network Mobility Signalling, Bell Labs Technical Journal 14(2) (2009), 155-184. 
[18] H.S. Jo, Ch. Mun, J. Moon and J.G. Yook, Self-Optimized Coverage Coordination in Femtocell Networks, IEEE Transactions on Wireless Communications 9(10) (2010), 2977-2982.

[19] S.Y. Yun and D.H. Cho, Traffic Density based Power Control Scheme for Femto AP, In IEEE PIMRC, 2010, pp. 13781383.

[20] G. de la Roche, A. Valcarce, D. Lopez-Perez and J. Zhang, Access control mechanisms for femtocells, IEEE Communications Magazine 48(1) (2010), 33-39.

[21] Y. Choi and S. Choi, Service Charge and Energy-Aware Vertical Handoff in Integrated IEEE 802.16e/802.11 Networks, In IEEE INFOCOM, 2007.

[22] P. Fülöp, S. Imre, S. Szabó and T. Szálka, Accurate mobility modeling and location prediction based on pattern analysis of handover series in mobile networks, Mobile Information Systems 5(3) (2009), 255-289.

[23] P. Bellavista, M. Cinque, D. Cotroneo and L. Foschini, Self-Adaptive Handoff Management for Mobile Streaming Continuity, IEEE Transactions on Networks and Service Management 6(2) (2009).

[24] J. Martinez-Bauset, J.M. Gimenez-Guzman and V. Pla, Optimal Admission Control in Multimedia Mobile Networks with Handover Prediction, IEEE Wireless Communications 15(5) (2008).

[25] P. Stoica and J. Li, Source Localization from Range-Difference Measurements, IEEE Signal Processing Magazine 23 (6), 63-65, (2006).

[26] C. Yu, W. Xiangming, L. Xinqi and Z. Wei1, Research on the Modulation and Coding Scheme in LTE TDD Wireless Network, In ICIMA, 2009, pp. 468-471.

[27] K. Kobayashi and T. Katayama Analysis and Evaluation of Packet Delay Variance in the Internet, IEICE Transactions on Communications E-85B(1) (2002), 35-42.

[28] W. Daamen and S.P. Hoogendoorn, Free Speed Distributions for Pedestrian Traffic, presented at Transportation Research Board, 85th Annual Meeting, (2006).

[29] Small Cell Forum, Interference management in OFDMA Femtocells, (2010).

[30] 3GPP TS 36.300 v 10.6.0, Technical specification group radio access network; evolved universal terrestrial radio access (E-UTRA); physical channels and modulation, 2011.

[31] S. Sesia, I. Toufik and M. Baker, LTE-the UMTS long term evolution: From Theory to Practice, West Sussex: Wiley, 2009.

Z. Becvar received the MSc and PhD degree in telecommunication engineering from the Czech Technical University in Prague, Czech Republic in 2005 and 2010 respectively. Currently, he is working as the assistant professor at the Department of Telecommunication Engineering at the same university. He was involved in Sitronics and Vodafone R\&Ds in Prague, Czech Republic. $\mathrm{He}$ is a member of more than 15 program committees of international conferences and journals and he published more than 50 papers in international conferences or journals and 3 book chapters. Further, he participates in several projects founded by European Commission and in several national research grants. His current research interests include mobility support and radio resource management in future mobile networks with small cells.

P. Mach received his MSc and PhD degree in telecommunication engineering from Czech Technical University in Prague, Czech Republic in 2006 and 2010 respectively. During his study he joined research groups at Sitronics and Vodafone R\&D centers focusing on wireless mobile technologies. He has published more than 40 papers in international conferences or journals. He has been actively involved in several national and international projects. He participated in several projects founded by European Commission such as FIREWORKS, ROCKET, FREEDOM or TROPIC. His research interests include MAN/LAN networks implementing relays and small cells. He is dealing with aspects relating to radio resource management and cross layer optimization processes. 

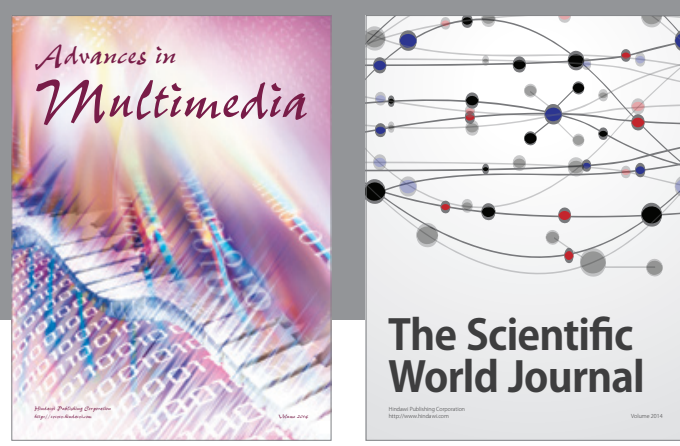

The Scientific World Journal
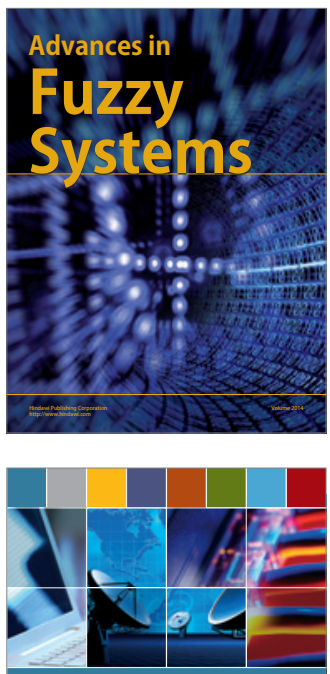

Computer Networks and Communications
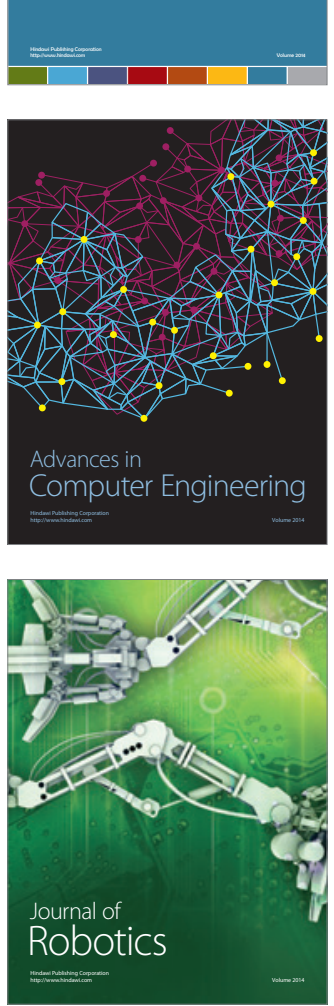
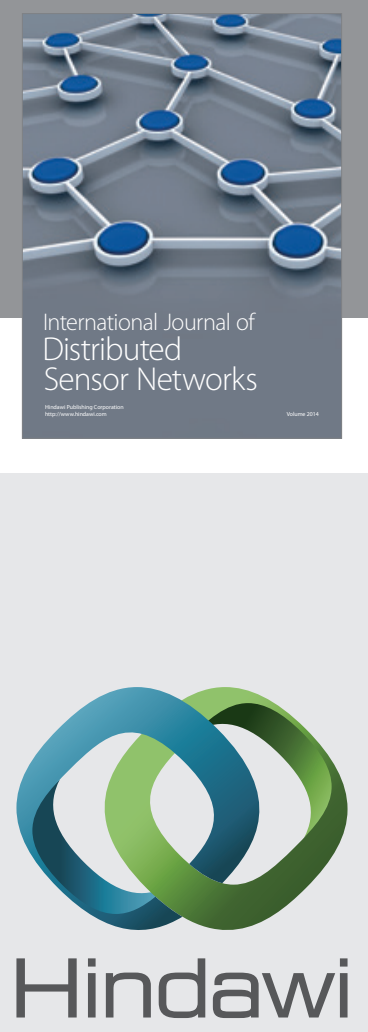

Submit your manuscripts at

http://www.hindawi.com
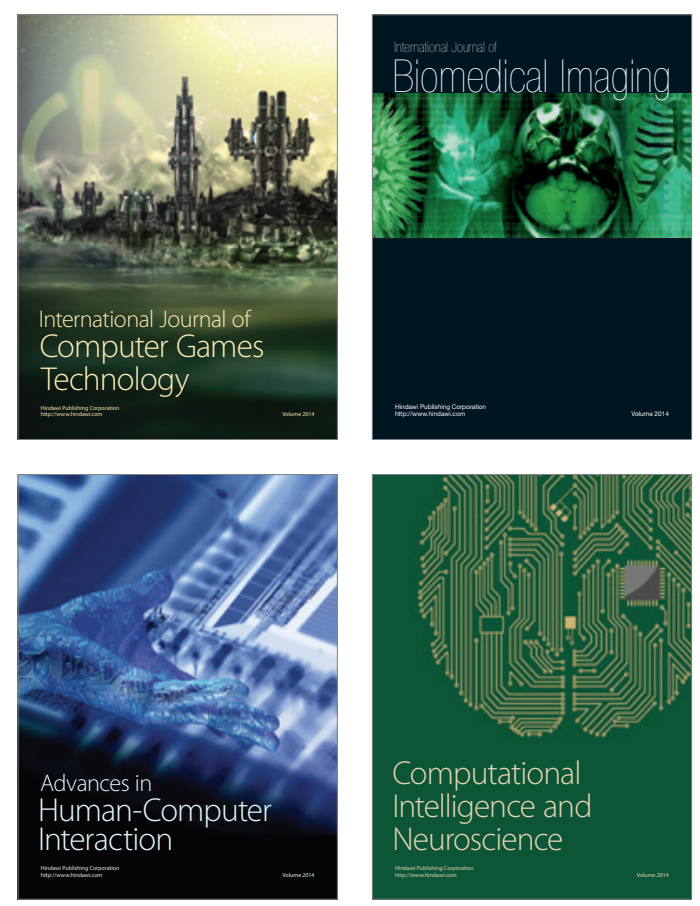
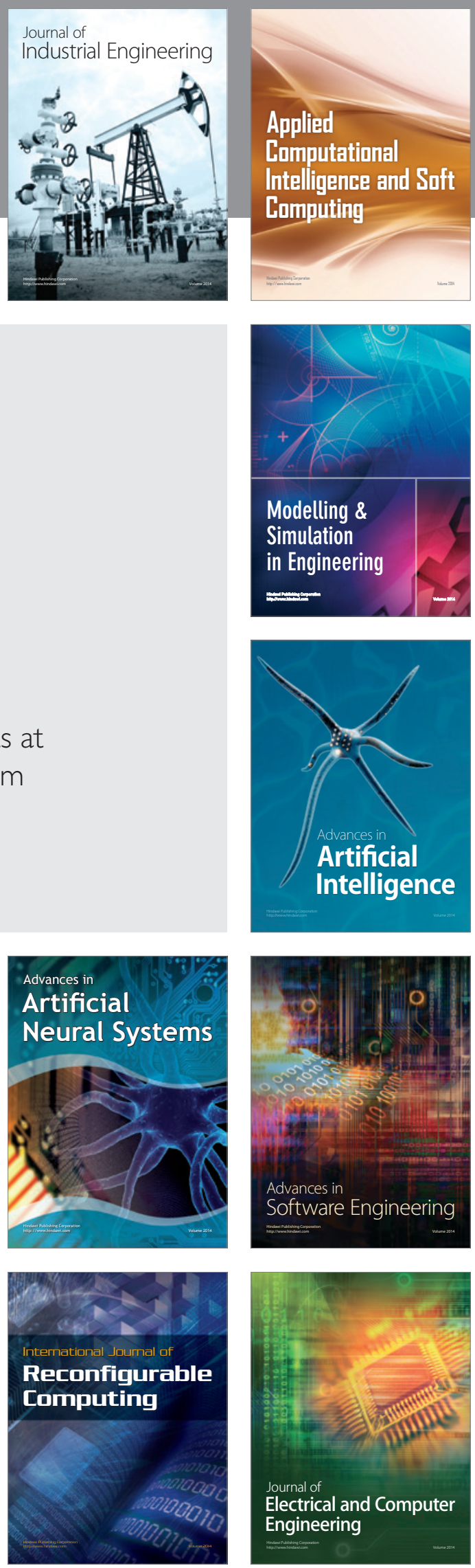